\title{
Peptide conjugated multipurpose carbon dots as nano-cargoes for glioblastoma brain tumors
}

Target specificity and the effective translocation of nano-cargoes into the tumor cells are still challenging. This study is an effort to build up a dual drug, dual peptide tethered quadruple nano-cargo, which specifically binds to the receptor IL-13R $\alpha 2$, which is highly expressed on high-grade gliomas compared to normal brain tissue. Moreover, the nucleus targeted peptide (InPep-1) enhances the DNA annihilation of chemotherapeutics' efficacy by delivering them to the nucleus. The conjugation of each peptide and drug on carbon dots (C-dots) was confirmed by the analytical spectroscopic methods of UVVis, fluorescence, FTIR, and MALDI-TOF.

The in vitro cell studies proved the efficacy of the quadruple conjugate, which reduced the cell viability to $8.8 \%$ at the concentration of $1 \mu \mathrm{M}$ in SJGBM2. The receptor expression study of the cell lines and the subsequent cell viability experiment further exhibited the efficiency of the quadruple conjugate, which showed the lowest cell viability in SJGBM2 and U87 that had relatively higher levels of receptor expressions.

However, the PKCHA and KNS42 displayed an inverse behavior with the receptor expression level and the viable cell count. The highest receptor level expressed KNS42 displayed a highest cell viability percentage even though we expected that to show up lowest cell viability. On the other hand, the least level of receptor expressed PKCHA exhibited a lowest cell viability percentage even though we expected that to display a highest cell viability percentage. This inverse behavior can be explained by two hypotheses: 1) Even though the PKCHA displayed least number of receptors, by limiting the nanoparticle uptake, it might be highly sensitive to the two chemotherapeutic agents to display a lowest cell viability percentage. 2) Even though the KNS42 expressed higher number of receptors, the number of active binding sites for the shPep-1 might be lower, which limits the nanoparticle uptake. Thus, the receptor binding study is yet to experimented to understand the above hypotheses. 